\title{
Datos bibliométricos y cienciométricos de Acta Pediátrica de México
}

\section{Journalmetrics of Acta Pediátrica de México.}

En los últimos 3 años, Acta Pediátrica de México (APM) ha reconfigurado su proceso de gestión editorial, pero la esencia de APM continúa siendo la misma que hace 37 años propusiera el Comité Editorial presidido por el Dr. Jorge Espino Vela: difundir los resultados de la labor asistencial, docente y de investigación de nuestras instituciones de salud, y en beneficio de la niñez mexicana.

En la última década, APM ha dejado de ser una revista solamente institucional, y se ha convertido en un referente de la Pediatría latinoamericana. Por tal motivo, es indispensable que APM se fortalezca y cumpla con las exigencias editoriales de un mundo digital donde la información fluye de manera inmediata.

Existen diversos indicadores para clasificar una revista de acuerdo a su calidad de contenido y el impacto de sus publicaciones, mismos que tienen virtudes y deficiencias que han sido discutidas en la literatura en múltiples ocasiones. Independientemente de las características de tales indicadores, los que editamos revistas científicas los vemos como parámetros de comparación y focos de atención para dirigir nuestros esfuerzos de mejoramiento continuo.
Por tal motivo, en esta Editorial comparto la información de los primeros resultados de los indicadores bibliométricos y cienciométricos de APM, así como la forma en la que se calculan para su mejor entendimiento. La intención de esto es compartirles un punto objetivo de comparación para el futuro de APM, así como reforzar la invitación a colaborar con nosotros publicando los resultados de sus investigaciones, asegurándoles que APM es una de las plataformas científicas más leídas en Latinoamérica.

La pregunta más frecuente que nos externan nuestros lectores y colaboradores es con respecto al factor de impacto (FI) de APM. El más conocido de estos es el que otorga el Institute for Scientific Information (ISI) mediante el Web of Knowledge (WOK) Journal Citation Reports (JCR), que estuvo manejado muchos años por Thomson Reuters, y actualmente está bajo la administración de Clarivate analytics. Este ISI-WOK-JCR tiene distintas bases de datos, colecciones o índices [e.g. ScienceCitationIndex (SCI), Social Science Citation Index, Arts \& Humanities Citation Index).

En términos generales el Fl se calcula anualmente de la siguiente forma: 
Ejemplo para el factor de impacto del año 2016: (Figura 1).

$$
F I 2016=\frac{A C 2015+A C 2014}{A P 2015+A P 2014}
$$

Figura 1. Cálculo del factor de impacto (FI) en 2016. AC: artículos citados; AP: artículos publicados.

APM está considerada en la base de datos Emerging Sources Citation Index (ESCl) junto con otras 5,720 revistas, lo que significa que está siendo evaluada para su incorporación en el $\mathrm{SCl}$ del ISI-WOK-JCR; al lograr esto, APM tendrá un FI.

Desde el año 2015 APM fue incorporada al Índice de Revistas Mexicanas de Investigación Científica y Tecnológica del Consejo Nacional de Ciencia y Tecnología (CONACyT). Dicho índice cuenta con 2 categorías y 8 grupos [i.e. Categoría 1 - Revistas indizadas en Scopus o WoS: grupos Q1, Q2, Q3, o Q4, Categoría 2 - Revistas no indizadas en Scopus o WoS: grupo de competencia internacional $(\mathrm{RCI})$, de competencia nacional (RCN), en consolidación (REC), o en desarrollo (RED)]. APM está considerada en el Q4, lo que le ha permitido aplicar a diversas convocatorias CONACYT.

APM está indizada en Google Académico. Google usa el índice h para dar su clasificación y se calcula de la siguiente forma: una revista tiene un índice $\mathrm{h}$ de $\mathrm{X}$ si esa revista ha publicado $X$ artículos citados al menos $X$ veces. Ejemplo: si APM publica 5 artículos, y cada uno es citado 21, 10, 7, 4 y 2 veces, el índice h corresponderá a 3 (porque sólo 3 de los 5 artículos recibieron al menos 5 citas). Google considera únicamente las publicaciones más citadas de la revista para calcular una base del índice h (h-core). Es decir, Google sólo tomaría en cuenta los 3 artículos que fueron citados 21, 10 y 7 veces, y reportaría la mediana de este h-core (i.e. 10). Al considerar dichos datos a lo largo del tiempo, Google reporta un índice h5 y la mediana del índice h5 tomando en cuenta los últimos 5 años. APM tiene un índice h5 de 8 y una mediana del índice h5 de 9. De manera comparativa, Pediatrics cuenta con índice h5 de 107 con una mediana de 163, ocupando el primer lugar dentro de la categoría Health \& Medical Sciences - Pediatric Medicine.

Scopus Scimago Journal \& Country Rank (SJR) considera a APM en el cuartil 4 (Q4) con un índice $h$ de 1 , indicador SJR de 0.119. Este último indicador es el promedio del número de citas generadas en un año dado, dividido entre el total de las publicaciones de los 3 años previos. De acuerdo a este sistema de clasificación, APM ocupa la posición 56 de 101 revistas mexicanas, 14 de 28 revistas mexicanas de medicina, y 2 de 3 revistas mexicanas de pediatría incluidas en SJR. SJR permite tener un visualizador de sus indicadores, mismo que hemos incorporado a nuestra página principal (www.actapediatrica. org.mx) y cuya imagen aparece de la siguiente forma: (Figura 2).

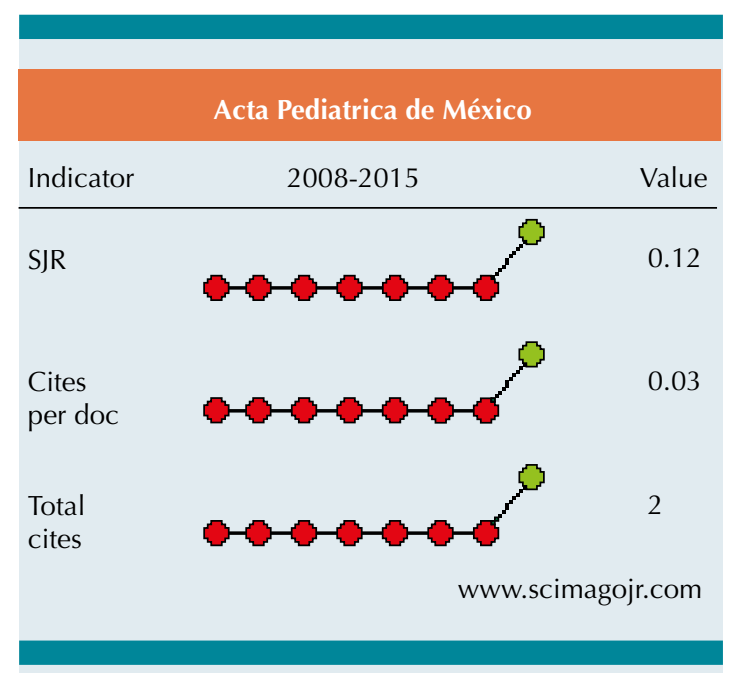

Figura 2. Visualizador de indicadores SJR. 
Scopus también reporta Source Normalized Impact per Paper (SNIP) de 0.068 para APM. Este indicador, ajusta las citaciones de las publicaciones por el potencial de citación dentro del contexto temático y de manera opcional por región (i.e. medicina, pediatría, México) y nos ubica en la revista 244 de 256 incorporadas bajo la clasificación "Pediatrics, Perinatology and Child Health".

APM también está indizada en Scientific Electronic Library Online (SciELO) y para el período 2014-2015 calculó un factor de impacto de 0.0211 (otorgado por 2 citas de 95 publicaciones realizadas en dicho período).

Finalmente, APM ha estado en Latindex desde 1980 en su versión impresa y desde 2010 en su versión digital, y cumple satisfactoriamente con 33 de 33 y 34 de los 36 estándares de calidad respectivamente, haciéndonos falta meta-etiquetas y servicios de valor añadido en la versión digital, mismas que están en proceso de incorporación.

Los datos bibliométricos y cienciométricos descritos en esta Editorial son incipientes y no reflejan lo que ha ocurrido con nuestras publicaciones a lo largo de la historia de APM. Se requiere de tiempo para que dichos datos reflejen el verdadero impacto de nuestras publicaciones en la comunidad científica internacional.

Es importante mencionar que ninguno de estos indicadores toma en cuenta la trascendencia de APM en la difusión de conocimientos clínicos y científicos a la comunidad de profesionales de la salud dedicados al componente asistencial de la pediatría mexicana y latinoamericana, quienes tradicionalmente han representado nuestra principal audiencia. APM tiene interés en expandir el horizonte de sus alcances e incrementar la audiencia y colaboración de investigadores clínicos y básicos activos. Al contar con mayor participación de dicha audiencia incrementaremos significativamente la citación de nuestras publicaciones y consecuentemente mejoraremos nuestros indicadores.

APM tiene el compromiso de continuar siendo un referente de la pediatría latinoamericana y ascender en la desafiante escala de los indicadores bibliométricos y cienciométricos, sin perder de vista el propósito fundamental de beneficiar a la niñez.

\section{Sitios recomendados}

http://wokinfo.com/products_tools/multidisciplinary/esci/

http://www.revistascytconacyt.mx

https://scholar.google.com.mx/citations?view_ op=top_venues \&hl=es

http://www.scimagojr.com/

https://www.scopus.com/sources?zone=\&origin =sbrowse

http://132.247.70.65/latindex/inicio

Dr. Armando Partida Gaytán Coeditor Acta Pediátrica de México dr.partida.g@gmail.com 